\title{
Measuring Reflective Power with the Eye
}

\author{
Xiang Chen*
}

\begin{abstract}
Although the legitimacy of using the eye as an essential instrument in photometric experiments had been questioned by critics, the practitioners of visual photometry in the 18th and 19th centuries were convinced that the eye was reliable and capable of making accurate judgments in comparing brightness. They demonstrated their belief through their efforts in searching for the optimal conditions for the eye in photometric measurements. Eventually, they were able to measure reflective power with accuracy comparable to today's standards by developing a body of practice, including both instrumental designs and experimental procedures, which aimed at maintaining the eye's sensibility in brightness comparison.
\end{abstract}

Key words: Visual photometry; photometric instruments; reflective power; Fresnel coefficients; Pierre Bouguer; Johann Heinrich Lambert; William Herschel; Richard Potter; John Conroy; Lord Rayleigh.

\section{Introduction}

Visual photometry, the science of comparing brightness by directly using the eye, first appeared around the middle of the 18th century. Printed in 1760, Pierre Bouguer's Optical Treatise on the Gradation of Light was the first major publication in which the instruments and procedures of visual photometry were discussed systematically. In the next few decades, visual photometry developed rapidly - new photometric equipment such as photometers and light sources were invented, and a large number of photometric phenomena, including the illuminating power, transparency, and reflective power of various materials were investigated.

Despite its initial successes, visual photometry encountered strong criticisms. Some critics even questioned the legitimacy of the discipline. They believed that visual photometry was fundamentally faulty, because it employed the eye as an essential instrument for brightness matching. It was common knowledge that the eye could be extremely unreliable. As pointed out by John Herschel in 1827, one need only consider "the opening of the pupil, which admits the light, being continually expanding and contracting by the stimulus of the light itself, and the sensibility of the nerves which feel the impression varying at every instant." 1 The eye was always in a state of fluctuation, and so was its reliability in comparing brightness. According to these critics, accurate measurements of photometric phenomena could be achieved only by a physical approach, which substituted for the eye an instrument such as a thermometer to measure illumination. ${ }^{2}$

* Xiang Chen is associate professor of philosophy at California Lutheran University. His research is in history and philosophy of science. 
Despite these criticisms, visual photometry survived and prevailed. Over the 19th century, it continued to improve its performance in accuracy. By the end of the 19th century, it was able to offer measurements with an accuracy comparable to today's standards, and photometric data were widely used in physical optics to test optical theories of metallic reflection and to calibrate such optical constants as refractive indices and absorption coefficients. The development of visual photometry raises an interesting question: Given that visual photometry used the eye as an essential instrument, which, as pointed out correctly by its critics, is unstable in judging brightness, how could visual photometry achieve its success in accuracy? To answer this question, we must examine the causes of visual photometry's earlier failure and the reasons for its later success. In the following sections, I will review the development of visual photometry in its early years, focusing particularly on measurements of reflective power. Historical analysis will show that the poor performance of visual photometry in its early years was not because the eye was used as an essential instrument in brightness matching, but resulted mainly from inappropriate uses of the eye. The later success of visual photometry stemmed from the efforts of several generations, and was embodied in a series of new instrumental designs and experimental procedures used to search for the optimal conditions for the eye in brightness comparison.

\section{The Principle of Simultaneous Comparison}

Pierre Bouguer (1698-1758), royal professor of hydrography, laid the foundation of visual photometry in the early 18th century. Bouguer (figure 1) was intrigued originally by the problem of determining the intensity of light from various celestial subjects, and in 1725 he succeeded in determining the intensity of moonlight by comparing its light to that of a candle. Bouguer's contribution to visual photometry was essential. Although all of his photometric measurements turned out to be inaccurate by today's standards, many experimental procedures that he proposed, particularly the principle of simultaneous comparison, were sound and dominated the practice for the next two hundred years.

One of the earliest attempts to determine the intensity of light was by Christiaan Huygens, who in the 17th century had tried to compare the brightness of the sun with that of the star Sirius. Huygens used a long tube with an adjustable aperture at the far end as his instrument. To make the comparison, he first pointed the tube at the sun and observed a diminished sunlight by reducing the size of the aperture. He then waited until the evening and pointed the tube at the star. By adjusting the size of the aperture, he obtained starlight with the same brightness as the illumination of sunlight. The ratio of the intensity of the sun to that of the star was in inverse proportion to the ratio of the areas of the two openings. According to Bouguer, Huygens's procedure was fundamentally flawed. The main problems were that the eye, an extremely delicate organ, has different levels of sensibility during the course of a day and responds differently to the same level of brightness. Even worse, no one could remember brightness even after a very small interval of time, so that comparing the brightness of sunlight to that of starlight when observed at 
different times was extremely unreliable. Bouguer insisted that "we can only judge directly the strength of the two sensations when they affect us at the same instant."3 In other words, visual comparisons must be made simultaneously. Comparison of the intensities of sunlight and starlight, for example, could only be achieved in two steps - first to compare sunlight (or starlight) with an auxiliary light, and then to compare the auxiliary light with starlight (or sunlight).

Another aspect of the principle of simultaneous comparison concerned the subjects to be compared. Before Bouguer, a variety of parameters had been used in the comparison of illuminations. For example, Anders Celsius in the early 18th century had used degree of distinctness as the subject of comparison in his photometric measurements. He had placed two small objects at different distances and adjusted the intensity of light falling on each one until he saw both of them with the same degree of distinctness, and he then calculated the ratio of the intensities according to the ratio of the distances. According to Bouguer, Celsius's procedure also was problematic because the estimation of distinctness was highly subjective. People with different visual problems, such as far-sightedness or nearsightedness, could have different judgments regarding the distinctness of an object. To avoid arbitrary decisions, Bouguer suggested that brightness should be the only parameter used in photometric comparisons. "As we are considering only the

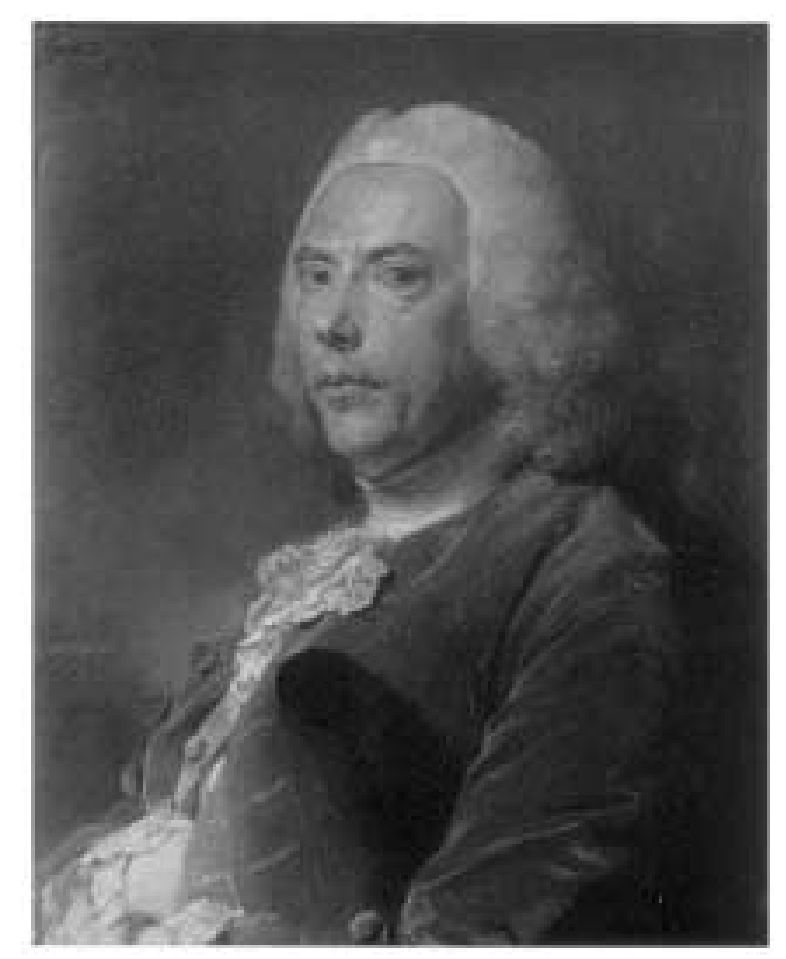

Fig. 1. Pierre Bouguer (1698-1758). Source: Pierre Bouguer, Optical Treatise on the Gradation of Light, translated by W. E. K. Middleton. (C) 1961 The University of Toronto Press. 


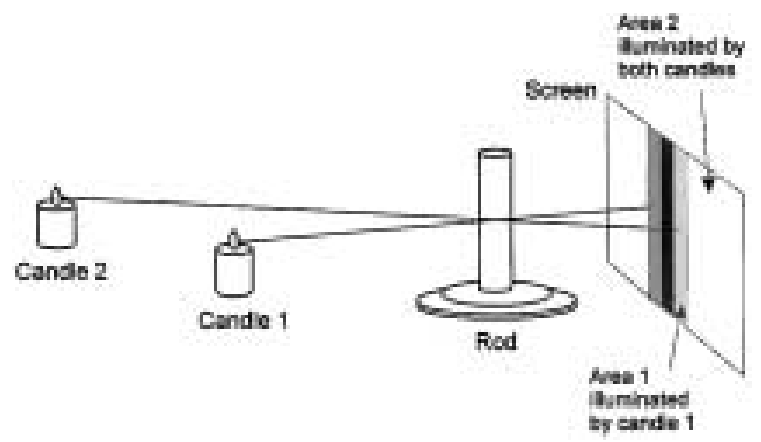

Fig. 2. Bouguer's apparatus for determining the sensitivity of the eye.

amount of light or its brightness, it does not matter whether the observer has long or short sight, good sight or bad. If the rays cross before having reached the retina or if they come together farther back, nevertheless they act on the back of the eye. There is nothing lost, and the total effect is always the same as regards the intensity of the impression." 4

The principle of simultaneous comparison thus defined the role of the eye in photometric measurements. According to Bouguer, the eye should be used only as a null indicator to mark the equality of brightness of two luminous bodies, rather than to estimate the degree of illumination. Using the eye as a null indicator reflected that the eye was extremely sensitive and reliable in judging equality of brightness. Bouguer designed an experiment to demonstrate this capacity of the eye. His apparatus included a white screen, two identical candles, and a rod placed between the screen and the candles, casting two shadows on the screen (figure 2). Bouguer set one of the candles at a distance of one foot from the screen and slowly increased the distance of the other candle until its shadow disappeared, that is, until the difference in brightness between Area 1 and Area 2 became invisible. After repeated experiments, he found that the shadow of the second candle always remained visible when its distance from the screen was less than eight feet, but it disappeared when the distance was about eight feet. These results suggested that two illuminated areas became visually indistinguishable when the difference in their brightnesses was smaller than $1 / 64$ (that is, $1 / 8^{2}$ ), or about $1.5 \%$. In other words, the eye was able to distinguish differences in brightness as small as $1.5 \%$ (later this quantity was called the brightness-difference threshold).

Following the principle of simultaneous comparison, Bouguer constructed various photometers to compare the brightnesses of different light sources and to measure the optical properties of various materials, including their transparency and reflective power. Bouguer's photometers can be divided roughly into two types: one type was built on the inverse-square law of distance, that is, the brightness level in the matching fields was adjusted by moving the light sources. The other type used the sizes of light sources as the operative parameter, that is, the brightness level was adjusted by changing the size of the light sources. 
In his measurements of reflective power, Bouguer mainly used the second type of photometers. One was made simply of two pieces of cardboard, one of which served as a screen, the other as a partition, controlling the amount of light from the light sources (figure 3). There were two adjustable apertures in the partition. The reflecting material, either a mirror or a liquid such as water or mercury, was placed horizontally between the partition and the screen, so that it reflected the light from aperture $P$ to hole $R$ in the screen, which served as one of the matching fields. A beam of direct light from aperture $Q$ was directed to hole $S$, the other matching field. Several black curtains were placed between the apertures and between the reflecting material and screen to avoid interference between different light beams. After carefully adjusting the positions of the partition and screen to make certain that the distances traversed by the reflected and direct light were equal, Bouguer adjusted the sizes of the apertures until equal brightnesses appeared in the matching fields. The reflecting power of the material was calculated in terms of the ratio of the sizes of the two apertures. ${ }^{5}$

Using this photometer, Bouguer measured the reflective power of mercury, water, metallic mirrors, and various kinds of glass. Although Bouguer's measurements were built upon a sound principle, his results were inconsistent. On one occasion, he reported that the reflective power of water at an angle of incidence of 76.5 degrees was $21.3 \%$, but on another occasion, the same measurement became $24.4 \%$, more than $15 \%$ higher than the previous value. ${ }^{6}$ In hindsight, most of Bouguer's measurements carried double-digit relative errors, compared to currently accepted measurements or theoretical calculations. For example, he reported that the reflective power of plate glass at 0 degrees (normal incidence) was $2.5 \%$, about $40 \%$ lower than the currently accepted value. His measurements of the reflective power of water, $1.8 \%$ at 0 degrees and $72.1 \%$ at 89.5 degrees, were 10 to $20 \%$ lower than the currently accepted values.*

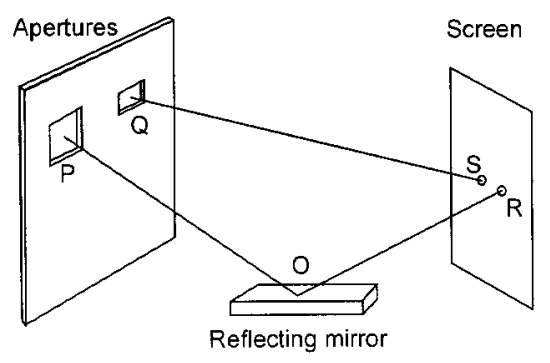

Fig. 3. Bouguer's photometer.

* The currently accepted values of the reflective power of transparent materials are calculated using the Fresnel formula:

$$
R=\frac{1}{2}\left[\frac{\sin ^{2}\left(\theta-\theta^{\prime}\right)}{\sin ^{2}\left(\theta+\theta^{\prime}\right)}+\frac{\tan ^{2}\left(\theta-\theta^{\prime}\right)}{\tan ^{2}\left(\theta+\theta^{\prime}\right)}\right]
$$

where $\theta$ is the angle of reflected light and $\theta^{\prime}$ the angle of refracted light $\left(\sin \theta^{\prime}=\sin \theta / n\right.$, where $n$ is 
Some of Bouguer's errors in measurement probably resulted from negligence. For example, when he measured the reflective power of glass, Bouguer did not consider the effect of the reflection from the second surface, which could enhance the intensity of the reflected light. Other errors might have resulted from the limitations of his experimental conditions. In his measurements for liquids, for example, Bouguer found that the surface of a liquid was not perfectly plane but convex, especially when the containing vessel was not sufficiently large. The convexity of the surface weakened the intensity of the reflected light, which in hindsight explains why most of Bouguer's measurements for liquids were lower than the currently accepted values.

Bouguer also made some compromises in his measuring process to maintain the integrity of his experimental design. For example, to make certain that the lengths of the direct and reflected beams were equal, Bouguer had to rotate the screen to a certain angle and thus exposed his eyes directly to one or both light sources. It seems that he did not take any steps to eliminate direct light, which quickly might cause eye fatigue by creating a strong contrast with the dark background. Furthermore, to make certain that the direct beam and part of the reflected beam ( $P O$ in figure 3) were parallel so that they came from two parts of the sky with equal elevation, Bouguer had to place the reflecting material about seven to eight feet from the apertures, which made the adjustment of the apertures very difficult. Because he did not use an assistant or remote-control device, Bouguer was not able to adjust the sizes of the apertures constantly while maintaining continuous observation. Without constant adjustments of the level of brightness in the matching fields, as we will see in the next section, the sensibility of the eye decreases significantly.

\section{The Method of Constant Adjustments}

Photometry developed rapidly in the second half of the 18th century on the foundation laid out by Bouguer. His first successor was the French mathematician Johann Heinrich Lambert (1728-1777). In a book published in $1760,{ }^{7}$ Lambert outlined several fundamental laws of photometry, including the cosine law of illumination, the cosine law of emission, and the law of addition of illumination.* Lambert also designed a photometer, but it is unclear if he actually constructed it.

Major improvements in the measurement of reflective power were achieved by William Herschel (1738-1822). His need to evaluate the performance of telescopes triggered Herschel's photometric research. By the late 18th century, it was common

the refractive index). For plate glass $n=1.515$, its reflective power at zero degrees should be 0.04193 ; for water $n=1.33$, its reflective power at 0 and 89.5 degrees should be 0.02006 and 0.9465 , respectively.

* The cosine law of illumination states that the illumination of a surface is proportional to the cosine of the incident angle. The cosine law of emission states that the radiation from a single-unit surface is proportional to the cosine of the emission angle. The law of addition of illumination states that the illumination produced by multiple light sources is equal to the sum of the illumination produced by each source. 


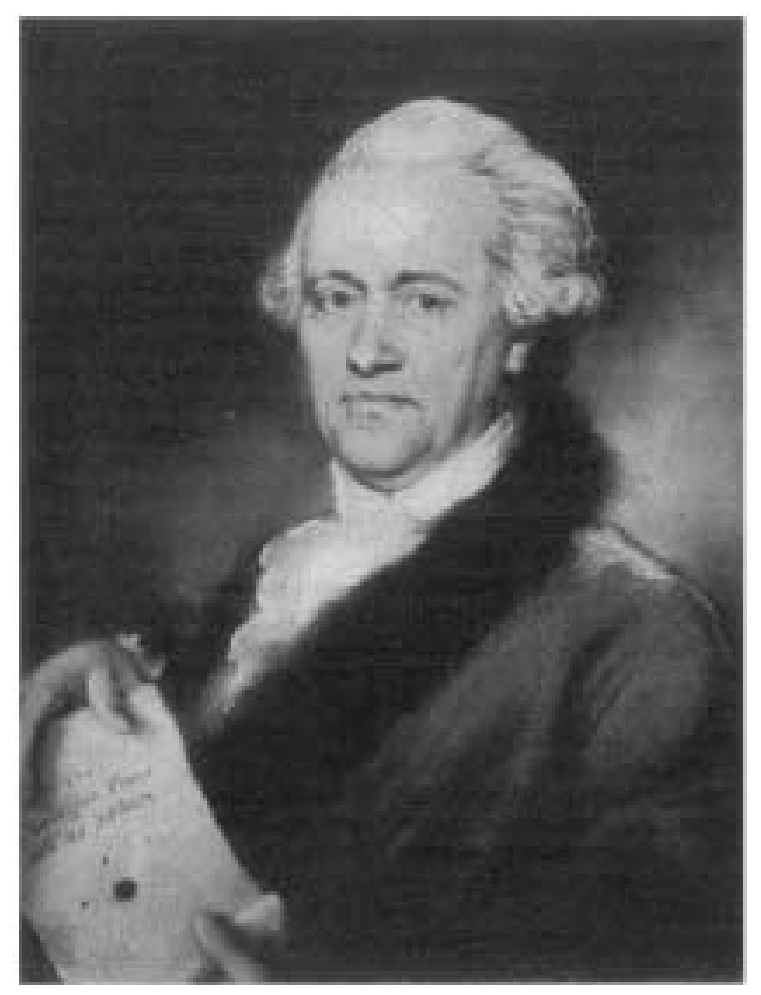

Fig. 4. William Herschel (1738-1822). Reprinted with permission of the Science Museum, London.

knowledge that, for the purpose of astronomical observations, magnifying power was not the only standard for evaluating telescopes. A good telescope should allow observers to detect faint objects regardless of its magnifying power. Herschel (figure 4) called this capacity "space-penetrating power," which depended upon three factors. The first was the aperture of the observer's pupil, which directly determined the amount of light reaching the retina. The second was the diameter of the concave mirror in a reflecting telescope or that of the objective lens in a refracting telescope. The third was the so-called illuminating power of the telescope, that is, the percentage of light transmitted through the optical system, which depended on the reflective power of metallic mirrors or the transmitting power of glass lenses. ${ }^{8}$

To determine the "space-penetrating power" of his own reflecting telescopes, Herschel conducted a series of photometric experiments around 1799 to measure the reflective power of the mirrors he used. Unlike Newtonian reflecting telescopes, Herschel's telescopes employed only a single convex mirror that reflected light perpendicularly, and the observer viewed the image by sitting in front of the telescope. Herschel therefore used a method that had been proposed by Bouguer to measure the reflective power of the mirror at zero degrees.

Figure 5 illustrates Herschel's experimental setup. He placed the mirror to be measured halfway between two identical reflecting screens, and a lamp somewhere 


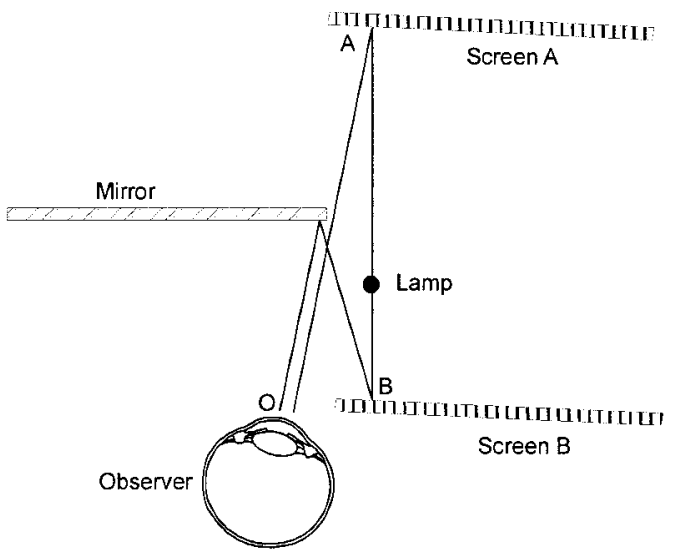

Fig. 5. Herschel's apparatus for measuring reflective power.

between the screens along line $A B$. From point $O$, he could see the image of the lamp in screen $A$ directly and the image of the lamp in screen $B$ after reflection from the mirror. He then adjusted the position of the lamp until the brightness of the direct and the reflected images appeared to be equal. Finally, he measured the distances of the lamp to the two screens and, according to the inverse-square law, calculated the reflective power of the mirror by means of the ratio of the squares of the distances.

Herschel used this procedure to determine the reflective power of the metallic mirror in his reflecting telescope, which was made of an alloy of $71 \%$ copper and $29 \%$ tin. After several experiments, he reported that, out of 100,000 incident rays, 67,262 were reflected by the mirror, ${ }^{*}$ that is, its reflective power was $67.262 \%{ }^{9}$ Although Herschel's instrument was relatively simple, his result was rather accurate. It is only $4.6 \%$ lower than the currently accepted measurement, and $2.6 \%$ higher than the value calculated from electromagnetic theory.**

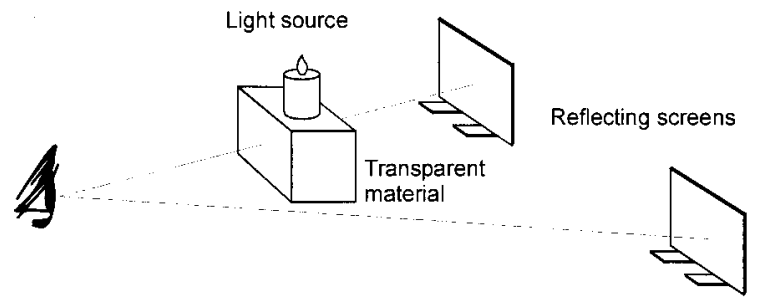

Fig. 6. Herschel's apparatus for measuring transparency.

* Notice that the five significance figures do not reflect the accuracy of the measurement, because they were obtained through calculation.

** The currently accepted measurement (0.0643) was given by Ernst Hagen and Heinrich Rubens at the beginning of the 20th century. The theoretical value is calculated according to electromagnetic theory from the following formula: 


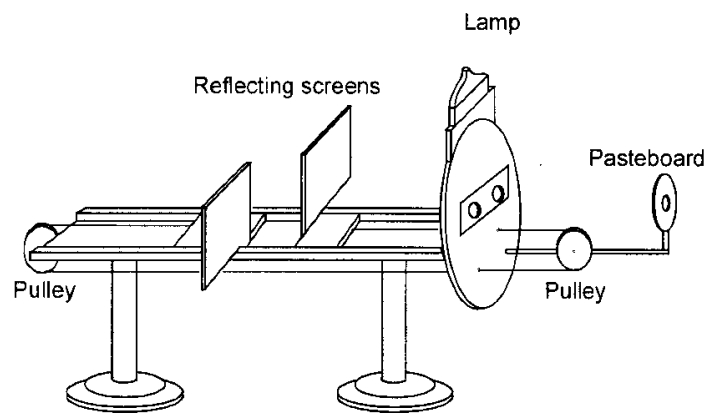

Fig. 7. Herschel's photometer.

Herschel's need to evaluate the "space-penetrating power" of refracting telescopes further motivated his investigations of the transparency of various materials. He first used a photometer that had been designed by Bouguer. Figure 6 illustrates Herschel's experimental setup. A candle was placed on top of the transparent material to be measured, illuminating two reflecting screens, which also served as the matching fields. One of the screens was viewed through the transparent material and the other directly. The distance to the latter reflecting screen was adjusted in the measuring process until the brightness of the two screens appeared to be equal. The transparency of the material then was calculated in terms of the ratio of the squares of the distances. But Herschel soon became unhappy with Bouguer's design. The uncontrolled light source (the candle) in Bouguer's photometer generated strong scattered light that was reflected from objects surrounding the instrument. The scattered light impinged on the two reflecting screens unevenly and thus affected the measurements. Herschel noted that "no other difficulty occurs in the execution of [Bouguer's] plan, than how to guard properly against the scatterings of the lamp: for the light which this will throw on every object, must not be permitted to come to the [reflecting screens]; since these scatterings cannot remain equal on both [reflecting screens], when one of them is moveable." 10 To overcome these defects, Herschel designed a new photometer.

The major components of Herschel's photometer (figure 7) also included a light source and two reflecting screens. Herschel placed the reflecting screens on two slides so that they could be moved easily along a board 14 feet long and 6 inches broad. A circular wooden screen was fixed to one end of the board, and there were two openings in its center. The transparent material to be measured, either glass or liquid, was placed in one of the openings. The light source was placed on top of the circular screen. About 3 feet beyond the circular screen there was a small piece of

$$
R=\frac{1}{2}\left[\frac{n^{2}\left(1+k^{2}\right) \cos ^{2} \theta-2 n \cos \theta+1}{n^{2}\left(1+k^{2}\right) \cos ^{2} \theta+2 n \cos \theta+1}+\frac{n^{2}\left(1+k^{2}\right)-2 n \cos \theta+\cos ^{2} \theta}{n^{2}\left(1+k^{2}\right)+2 n \cos \theta+\cos ^{2} \theta}\right]
$$

where $n$ is the refractive index, $k$ the absorption coefficient, and $\theta$ the angle of reflection. For a copper-tin alloy, $n=1.22$ and $k=2.7$ (for a wavelength $\lambda=6000 \AA$ ), so its reflective power should be 0.06907 . 
pasteboard with a hole in its center, through which the observer could compare the brightness of the two reflecting screens.

To overcome the difficulties caused by scattered light, Herschel replaced Bouguer's candle with a lamp inside a dark lantern. The lamp burned smoothly inside and leaked no light to the room. In the front side of the lantern, there was a hole covered with a spout 5 inches long. By these devices, Herschel ensured that the light from the source fell only on the reflecting screens, and in this way he practically eliminated any interference from scattered light.

Herschel's photometer also dealt with a problem directly related to an inherent aspect of the eye. Many practitioners in visual photometry had noted that, after they had viewed the matching fields for a while, they often were perplexed as to whether the match in brightness was real. This confusion occurs because the sensibility of the eye is low for small differences in brightness, and decreases quickly when the eye has viewed two almost equally illuminated surfaces too long. ${ }^{11}$ Through experience, Herschel realized that one way to maintain the sensibility of the eye was to produce a slight contrast between the fields to be matched by constantly adjusting the brightness of either or both during observation. In his photometer, Herschel utilized several new devices to make such constant adjustments while maintaining continuous observation. He fastened both reflecting screens to a string that passed over two pulleys placed at the ends of the broad. The string and pulleys functioned as "remote-control" devices, allowing Herschel to adjust the positions of the reflecting screens without leaving his seat. This method of constant adjustments was effective in maintaining the sensibility of the eye in comparing brightness and was adopted widely by later practitioners of visual photometry. When remote-control devices were unavailable, assistants often were employed to make constant adjustments during observation.

Using his newly constructed photometer, Herschel measured the transparency of various glasses and liquids. He placed the glass to be measured over one of the openings in the circular screen, left the other open, and adjusted the distances to the reflecting screens by pulling the string until they appeared to be of equal brightness. By measuring the distances to the two reflectors, he calculated the transparency of the glass according to the inverse-square law. His results show that the transparency of plate glass was $83.2 \%$, crown glass $79.7 \%$, flint glass $96.6 \%$, air $79.6 \%$, and seawater $71.2 \% .^{12}$

Herschel's measurements of transparency were more accurate than Bouguer's. For example, Bouguer had reported that six pieces of plate glass, in the experimental setup illustrated in figure 6 , eliminated $70 \%$ of the incident light. This amounts to a transparency of $55.5 \%$, almost $40 \%$ lower than the currently accepted value. However, Herschel's measurement of the transparency of plate glass at $83.2 \%$ is only about $10 \%$ lower than today's value, and his measurement of flint glass is only $3 \%$ higher.*

\footnotetext{
* To calculate transparency, one assumes that the absorption is minimum and the loss of light is caused only by the two reflections from both surfaces. Given that the refractive index of plate glass is 1.515 , its transparency should be $91.74 \%$; for flint glass the transparency should be $93 \%$.
} 
Based on these measurements, Herschel also reported "the proportion of light which is stopped by each of the substances," which he called the "stoppage of light," and which was the arithmetical complement of transparency. ${ }^{13}$ For plate glass, crown glass, and flint glass, the stoppage was $16.8 \%, 20.3 \%$, and $3.4 \%$, respectively. Since absorption by thin glass was negligible, reflections at the two surfaces are the only cause for loss of light. Reflective power $R$ can be derived from Herschel's "stoppage of light" $S$ according to the formula $R=1-\sqrt{1-S}$. Thus, Herschel's measurements of transparency implied that the reflective power of these glasses was $8.79 \%, 10.73 \%$, and $1.71 \%$, respectively. Herschel himself did not make these calculations, but by introducing the notion of "stoppage of light," he suggested a new method for determining the reflective power of a substance by deriving it from its transparency, instead of by measuring it directly. Herschel's indirect approach offered an alternative means for determining reflective power and generated some rather accurate measurements in the late 19th century.

\section{The Technique of Dealing with Contrast}

In the early 1830s, Richard Potter (1799-1868), Professor of Natural Philosophy and Astronomy at University College, London, made another attempt to measure reflective power. In his earlier years as an amateur scientist, Potter (figure 8) had

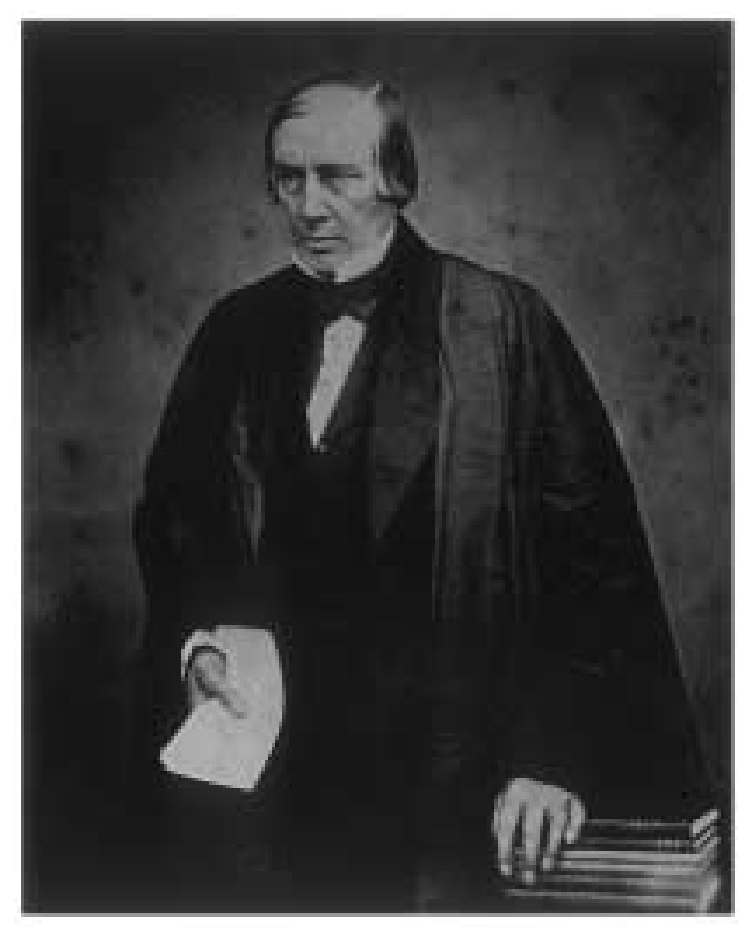

Fig. 8. Richard Potter (1799-1868). Courtesy of University College London. 


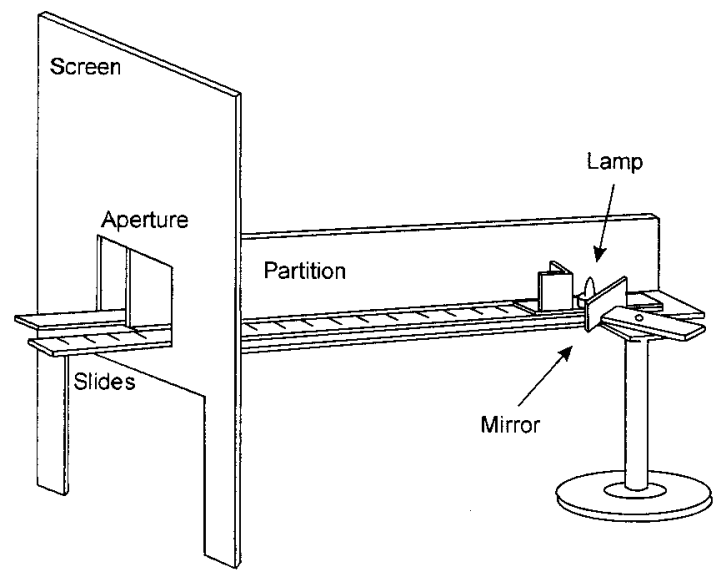

Fig. 9. Potter's reflecting photometer.

built a Newtonian telescope that used a concave metallic mirror to produce images and a small plane metallic mirror to reflect them off to the side for observation. His need to evaluate his telescope, specifically the reflective power of its mirrors at various angles, triggered Potter's photometric research. To measure the reflective power of mirrors at various angles of reflection, Potter constructed a reflective photometer in 1830 (figure 9).

The main components of Potter's photometer were an upright screen with an aperture and a horizontal board divided by a blackened partition. Two identical lamps were used, with each one fastened on the end of a slide on either side of the partition. To determine the reflective power at various angles, Potter added some special devices. He fixed the mirror to be measured to an arm that could be turned around an axis attached to the right-hand slide (figure 10). To intercept alternately the direct and reflected light, Potter installed two upright partitions perpendicular

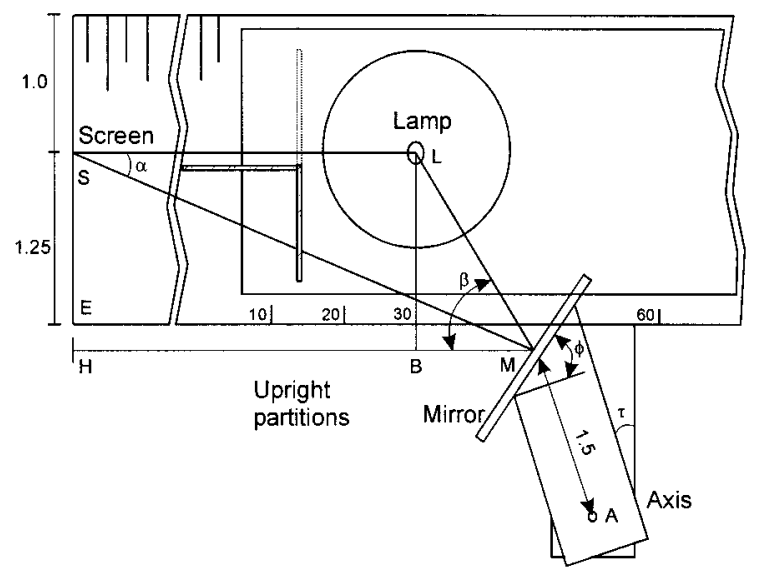

Fig. 10. Potter's reflecting photometer (details). 
to each other. When the partitions were in the direct-light position as shown in figure 10, they intercepted the reflected light; when the partitions were turned 90 degrees clockwise to the reflected-light position, they intercepted the direct light.

The major difficulty that Potter experienced in his experiments arose from "the fatigue of the eye experienced by looking long and intently at bright objects surrounded by darkness, which prevents it after some time judging accurately of very small differences [in brightness]." 14 This phenomenon is called contrast, which occurs when contiguous parts of the retina are stimulated very differently. Strong contrast can cause eye fatigue quickly and decrease significantly the eye's sensibility in comparing brightness. For example, a gray patch appears darker when viewed on a white background than it does when viewed on a black one.

To reduce contrast between the matching fields and the background, Potter covered the aperture with semi-translucent paper to decrease the brightness of the fields. He also invented several "remote-control" devices to enable him to conduct his experiments without exposing his eye to the direct light from the lamps. He put the lamps on moveable slides, and because their ends extended beyond the screen, he could adjust the positions of the lamps by pulling or pushing the slides while staying behind the screen. He marked the right-hand slide with divisions, in 0.25 inch intervals, so that he could determine the distance between the lamp and screen simply by reading off the divisions. By attaching strings to the corners of the perpendicular partitions, he also could turn them in either direction without leaving his seat.

Potter first measured the reflective power of several metallic mirrors, one composed of cast steel and the rest of copper-tin alloy. To begin with, Potter put the right-hand lamp and mirror in preset positions and turned the perpendicular partitions to stop the reflected light. He then made the first brightness match, adjusting the left-hand lamp until equal brightness appeared on the screen, and measuring the distance between the right-hand lamp and screen (the distance traversed by the direct light). Next he turned the partitions to stop the direct light and made a second brightness match by pulling the right-hand slide together with the lamp and mirror closer to the screen until equal brightness again appeared on the screen. He then measured the distance between the right-hand lamp and screen (the distance traversed by the reflected light). Finally, learning the distances traversed by the direct and reflected light, he calculated the reflective power of the mirror using the inverse-square law.

Among these operations, Potter's measurements of distances deserve our attention. His measurement of the distance traversed by the direct light was straightforward. He obtained this parameter simply by reading off the divisions on the slide. But Potter's method of measuring the distance traversed by the reflected light was peculiar. This parameter is the sum of the distance from the lamp to the mirror ( $L M$ in figure 10) and the distance from the mirror to the center of the aperture $(M S)$. The value of $L M$ was available before the experiment from the preset positions of the lamp and mirror, but the value of $M S$ was not, because the reflected light no longer fell onto the center of the aperture after the second brightness match. Potter made it clear that he actually did not measure MS. "It will be seen that the divisions commencing only at the thicker piece of wood, the 
distance of the lamp in the direct measurements, and the sum of the distances of the lamp to the mirror, and the mirror to the commencement of the divisions, must be added afterwards in the reflected ones," he said. ${ }^{15}$ In other words, Potter made an approximation by substituting for $M S$ the horizontal span between the mirror and screen $(M H)$, which was available by reading it off the slide. This was another measure that Potter took to reduce eye fatigue caused by contrast - if Potter had measured $M S$ directly, he would have exposed his eye to direct light from the lamps and the sensibility of his eye would have decreased quickly.

Potter's calculations also deserve our attention. Potter knew that if the light from the source did not fall onto a surface perpendicularly, then the illumination of the surface was proportional to the cosine of the angle of incidence $\alpha$. Since the reflected light was not perpendicular to the screen in Potter's experiments, the reflective power $P$ should have been calculated by using the following equation derived from Lambert's cosine law of illumination:

$$
P=\frac{1}{\cos \alpha} \times\left[\frac{D_{r e f}}{D_{\text {dir }}}\right]^{2},
$$

where $\alpha$ is the angle between the reflected light and normal to the screen, $D_{\text {ref }}$ the distance traversed by the reflected light, and $D_{d i r}$ the distance traversed by the direct light. But as for the distance $M S$, the angle $\alpha$ also had to be measured experimentally. For the same reason - to reduce eye fatigue - Potter made another approximation; he completely ignored the angle of incidence in his calculations.

These approximations, however, did not have any notable effect on Potter's measurements for metallic mirrors. Because metals have a relatively high reflective power, the right-hand lamp was still quite far away from the screen after Potter's second brightness match, usually more than 30 inches, and the angle of incidence $\alpha$ was close to zero degrees. These approximations caused only about a $0.1 \%$ deviation in his final measurements.

Potter reported that the reflective power of his copper-tin alloy mirror $(69 \%$ copper, $31 \%$ tin) was $67.5 \%$ at 10 degrees, $66.05 \%$ at 30 degrees, $65.07 \%$ at 50 degrees, and $65.16 \%$ at 70 degrees, and that the reflective power of his steel mirror was $58.86 \%$ at 10 degrees, $55.52 \%$ at 20 degrees, $54.13 \%$ at 50 degrees, and $54.67 \%$ at 60 degrees. The discrepancies between Potter's measurements and the values derived from electromagnetic theory are relatively small, mostly about $5 \% .{ }^{*}$

Potter soon turned his attention to the reflective power of glass. In his glass experiments, he used the same photometer and followed essentially the same procedures as those in his metallic-mirror experiments. In 1831, he published his measurements of the reflective powers of plate glass, crown glass, and flint glass at various angles of reflection, from 10 to 80 degrees. He reported that the reflective power of crown glass was $3.66 \%$ at 10 degrees, $4.17 \%$ at 30 degrees, $5.25 \%$ at 50 degrees, and $13.7 \%$ at 70 degrees.

* For copper-tin alloy, the refractive index $n=1.22$ and the absorption coefficient $k=2.7$ (for a wavelength $\lambda=6000 \AA$ ), so the reflective power should be 0.6907 at 10 degrees, 0.6901 at 30 degrees, 0.6859 at 50 degrees, and 0.6826 at 70 degrees; for steel $n=2.44$ and $k=1.36$ (for $\lambda=5800 \AA$ ), so the reflective power should be 0.5728 at 10 degrees, 0.5552 at 20 degrees, 0.5657 at 50 degrees, and 0.5582 at 60 degrees. 
Unlike metallic reflection, for which there was no theoretical account available in the early 1830s, reflection of transparent materials was well within the domain of the wave theory since the late 1820s. Based on the wave theory, Augustin Fresnel had deduced a formula for calculating the amount of light reflected by transparent materials (see footnote, page 443). Potter's measurements were all significantly lower than the theoretical values. According to Fresnel's formula, the reflective power of crown glass, for example, should be 0.0431 at 10 degrees, 0.0446 at 30 degrees, 0.0612 at 50 degrees, and 0.1755 at 70 degrees. In most cases, the discrepancies between Potter's measurements and the theoretical values are about $20 \%$. Potter realized the theoretical implications of his photometric measurements. Because of the discrepancies between his measurements and the theoretical values, he concluded that "if the formula [from] which they have [been] deduced from the undulatory hypothesis are found to give results at variance with observed phenomena, we are just entitled to draw an argument from it, against the hypothesis from which they emanated, as being also at variance with fact." 16

In his glass experiments, Potter used the same instrument and followed virtually the same procedure, but the accuracy of his glass measurements was much lower than that of his metallic measurements. One reason might be connected with the approximations he made in his measurements. The influence of these approximations was negligible in his metallic experiments, but their consequences became significant in his glass experiments. Because the reflective power of glass is low at small angles of reflection, Potter had to pull the glass mirror very close to the aperture in the second brightness match. When the angle of reflection was 10 degrees, for example, the glass mirror was only about 6 inches away from the aperture. In this position, the approximation he used for the reflected distance was about $2 \%$ lower than its true value, and the angle of incidence was about 13 degrees, which would generate errors as high as $8 \%$ in the final measurements. ${ }^{17}$

\section{The Requirement of Parallelism}

During the half century following Potter's experiments, no one made any further measurements of reflective power by the photometric method. This situation changed only in 1883 when Sir John Conroy (1845-1900) picked up the subject once again. Conroy conducted a series of photometric experiments to determine the reflective power of metals. His experimental design and procedure were in many ways similar to Potter's, but he made several significant changes. According to Conroy, Potter's photometer had a serious problem: "The two illuminated portions of the screen were not actually in contact, being separated by a dark shadow." By its very nature, the eye can detect reliably only differences in the brightness of adjacent surfaces. Thus, Conroy believed, Potter's design "must have interfered with the accuracy of the determinations." 18

Conroy reasoned that for the eye to make accurate judgments in comparing brightness, the gap between the matching fields had to be eliminated. To achieve this optimal condition, he constructed a new photometric head in which the matching fields were enclosed in a box with three apertures (figure 11). Light from 


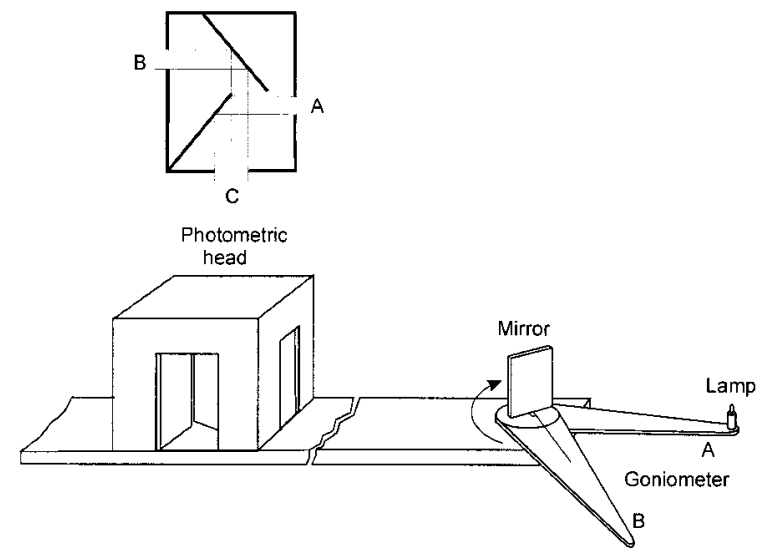

Fig. 11. Conroy's photometer.

two identical lamps placed at the opposite ends of a horizontal board (only one lamp is shown) entered the box through apertures $A$ and $B$, which the observer compared through aperture $C$. The matching fields, two pieces of white paper, were arranged in such a way that one was slightly in front of the other and overlapped it slightly. In this way, the two matching fields no longer were separated. When they were illuminated equally, the observer would see that the edge of the front paper vanished.

Another problem in Potter's experiments, according to Conroy, was his difficulty in determining the distance of the reflected light, which could cause eye fatigue rapidly. Conroy invented a new design that made measuring the reflected distance unnecessary. He attached a Babinet goniometer to the right-hand end of the horizontal board, with its axis on the center line of the board (figure 11). The right-hand lamp was fastened to the end of one of the arms of the goniometer (arm $A$ ). When the experiment began, Conroy set arm $A$ along the center line, adjusted the location of the left-hand lamp (not shown) until the matching fields appeared of equal brightness, and measured the distance from the left-hand lamp to the photometric head. Next, he placed the mirror to be measured vertically on a stage at the axis of the goniometer and perpendicular to $\operatorname{arm} B$, and he then rotated the entire goniometer until reflected light fell on the matching field. After adjusting the position of the left-hand lamp to bring about equal brightness, he measured its distance from the photometric head. Since the distance between the right-hand lamp and the matching field remained constant in the experiment, the reflective power of the mirror was calculated solely from the distance to the left-hand lamp, which could be determined easily by reading it off the scale attached to the board.

Using his new photometer, Conroy measured the reflective power of several metals at various angles. He reported that the reflective power of copper-tin alloy (69\% copper, $31 \%$ tin) was $66.13 \%$ at 10 degrees, $66.87 \%$ at 30 degrees, $67.26 \%$ at 50 degrees, and $67.65 \%$ at 70 degrees, and that the reflective power of steel was $54.38 \%$ at 10 degrees, $54.93 \%$ at 30 degrees, $56.74 \%$ at 50 degrees, and $58.09 \%$ at 
70 degrees. ${ }^{19}$ Although he employed a more sophisticated photometer, Conroy's measurements were about as accurate as Potter's. His measurements of copper-tin alloy, for example, carried relative errors between $2-3 \%$, and his measurements of steel had errors around 5\%.

A breakthrough in measuring reflective power occurred in 1886 when Lord Rayleigh (John William Strutt, 1842-1919) turned his attention to the subject. Rayleigh's photometric research was triggered by Ogden Rood, professor of physics at Columbia College, who had tested the Fresnel formula in 1870 by deriving reflective power from the transparency of glass. Rayleigh (figure 12) was dissatisfied with Rood's work, because he found that errors in measurements of transparency were "magnified" and transferred to measurements in reflective power. For example, although Rood's measurements of transparency carried relative errors as little as $0.3 \%$, the derived reflective power was almost $4 \%$ higher than the theoretical value. ${ }^{20}$ Thus, Rayleigh insisted that the Fresnel formula must be tested by direct measurements.

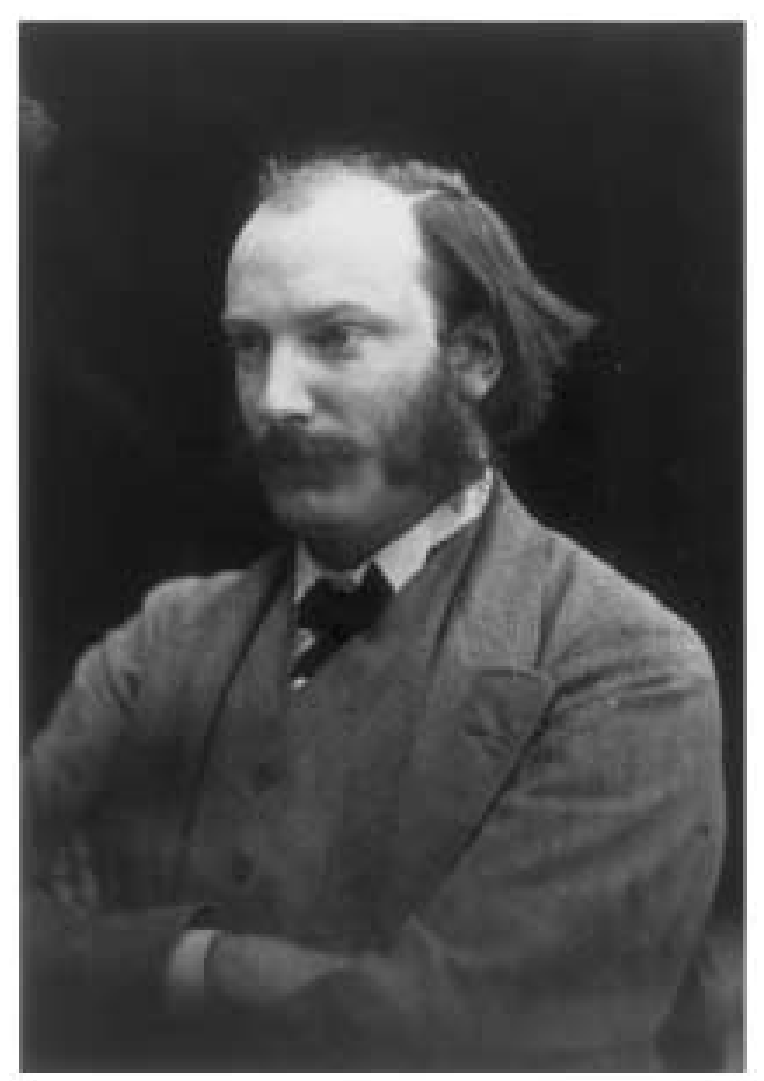

Fig. 12. Lord Rayleigh (John William Strutt, 1842-1919). Courtesy of the American Institute of Physics Emilio Segrè Visual Archives. 


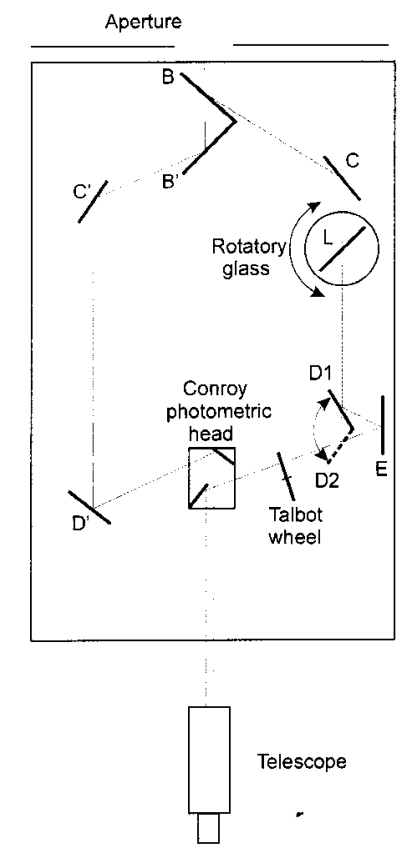

Fig. 13. Rayleigh's photometer.

Rayleigh constructed a new photometer to measure the reflective power of glass directly. Figure 13 shows its basic structure. The incident light first fell on the transparent plates $B B^{\prime}$. Through reflections the plates produced two beams of light of nearly equal intensity. The beam on the left-hand side functioned as the comparison light, which fell on a Conroy photometric head after being reflected twice by two silvered mirrors $C^{\prime}$ and $D^{\prime}$. To vary the relative brightness of the two beams, a glass plate $L$, capable of rotating about a vertical axis, was placed in the path of the right-hand beam. As the angle of incidence on $L$ increased, the brightness of the transmitted light decreased. After being transmitted through $L$, the beam could take two different paths. When the silvered mirror $D$ was in position 1 , the beam was further reflected by $E$, the glass to be measured. When $D$ was in position 2 , the beam reached the head after two reflections, exactly as did the left-hand beam. To compensate for the loss of light caused by reflection off $E$, a Talbot wheel was used when $D$ was in position 2 . The wheel consisted of a blackened disk of tin, from which a section of about $5 \%$ of its total area was cut out. When the wheel was rotating at a high speed (more than 25 revolutions per second), there was no perceptible flicker, and the transmitted light was of a stable, reduced brightness. ${ }^{21}$

Except for using the same photometric head, Rayleigh's photometer was quite different from Conroy's, but it addressed Conroy's same concerns. The first was the issue of distance measurements. To further overcome the difficulties caused by distance measurements, Rayleigh adopted an entirely new technique. He adjusted the illumination of the matching fields by rotating a piece of glass, and not by 
varying distances. This not only simplified the experimental process; it also improved the accuracy significantly, because errors in distance measurements would be magnified because of the inverse-square relationship between distance and brightness. However, adjusting the illumination by rotating a piece of glass had its own problem. To determine the proportion of light transmitted through the rotatory glass $L$ at various angles, Rayleigh used a table constructed by the Harvard astronomer Edward Pickering that was based on the same Fresnel formula that the photometer was intended to test. ${ }^{22}$ To avoid such a vicious circle, Rayleigh carefully chose the size of the opening in the Talbot wheel. When the Talbot wheel just compensated for the loss of light caused by reflection from the glass $E$, the rotatory glass plate $L$ could take nearly the same positions when taking the two sets of readings, with and without reflection from the glass $E$. In this way, Rayleigh believed that he could reduce the circularity in using Pickering's table to a minimum.

Another issue raised by Conroy was the relative positions of the matching fields. Conroy had realized that the two matching fields must be in contact, but according to Rayleigh, Conroy had addressed only part of the problem. The key issue was a requirement of parallelism, namely, that to achieve accurate comparison of brightness, all rays from the matching fields must be sensibly parallel. By eliminating the gap between the matching fields, Conroy made only a small part of the rays parallel. Furthermore, in practice the angle of view for each matching field should subtend not less than a degree at the eye for easy observation, which also produced non-parallel rays. Rayleigh's solution was to employ a telescope to enlarge the field of view without sacrificing parallelism. According to Rayleigh, when a telescope was employed, the maximum angular difference $\theta$ between rays from the two fields is given by

$$
\theta=\frac{(m a+b)}{r}
$$

where $a$ is the diameter of the pupil, $b$ the size of the matching fields, $m$ the magnifying power of the telescope, and $r$ the distance between the fields and the observer. Given that the size of the matching fields and the magnifying power of the telescope were fixed, there were only two ways to satisfy the requirement of parallelism: either by reducing the diameter of the pupil, or by increasing the distance between the matching fields and the observer. The first option, however, would diminish the brightness and consequently was unacceptable - the reliability of the eye in comparison of brightness depends sensibly on the brightness of the fields. ${ }^{23}$ Thus, Rayleigh had to take the second option. He placed his telescope more than 24 inches from the matching fields, and the overall size of his photometer was about 72 by 30 inches. That large scale caused many inconveniences: It no longer was possible for Rayleigh himself to make constant adjustments while observing (he had to employ an assistant), and it was difficult to find a level bed for all of the movable pieces of his photometer. But Rayleigh knew that it was necessary to meet these challenges, because the eye functioned better under these conditions. As he said, "the aperture of the eye controls the size of the apparatus." 24 
To begin a measurement, Rayleigh first set mirror $D$ in position 1 (to send the right-hand beam along its reflected path) and then adjusted the angle of the rotatory glass $L$ until the matching fields appeared to be equally bright. Next, he turned the mirror $D$ to position 2 (to send the right-hand beam along its direct path), introduced the Talbot wheel, and then adjusted the angle of the rotatory glass $L$ until the matching fields again appeared to be equally bright. After measuring the angular positions of $L$ in both settings, Rayleigh calculated the reflective power $R$ from the expression

$$
R=\frac{T_{D}}{T_{R}} \times W,
$$

where $T_{D}$ is the transparency of $L$ when the beam was sent along its direct path, $T_{R}$ is the transparency of $L$ when the beam was sent along its reflected path, and $W$ is the percentage of light transmitted by the Talbot wheel.

In his experiments, Rayleigh chose a prism of crown glass as his test material $E$, because he could get separate reflections from both surfaces of the prism. He recorded six observations, four by himself and two by his assistant. After taking their mean, he reported that the reflective power of crown glass was 0.04095 at $13^{\circ} 52^{\prime}$, about $10 \%$ lower than the value given by the Fresnel formula $(0.04514)$, which was a significant improvement compared to the values given by Potter. But unlike Potter who used the discrepancies to challenge the Fresnel formula, Rayleigh now used the Fresnel formula to calibrate his experiments, interpreting the discrepancy as an indication of imperfect measurements.

To make certain that his lower value of reflective power was real, Rayleigh gave his prism a thorough cleaning, and found that its reflective power increased to 0.04113. He further repolished the surface with putty powder, and achieved a much stronger reflection: the reflective power increased to 0.0476 , about $5 \%$ higher than the theoretical value. Rayleigh did not believe that the significant increase in reflective power he had achieved (almost 17\%) resulted entirely from the better-prepared surface of his prism. He suspected that part of the increase was caused by the rotatory glass $L$ and its associated Pickering table - the positions of the glass varied more than $10 \%$ in the two settings. To reduce the influence of the rotatory glass, Rayleigh enlarged the opening section of the Talbot wheel a little, and made another six measurements. This time he reported that the reflective power was 0.0452 , only $0.1 \%$ higher than the theoretical value. Based on these measurements, Rayleigh concluded that "the observed result now agrees remarkably well with that calculated from Fresnel's formula; ... recently polished glass surfaces have a reflecting power differing not more than 1 or $2 \%$ from that given by Fresnel's formula." 25

\section{Conclusions}

The failure of early visual photometry in making accurate measurements was not because the eye was used as an essential instrument in brightness matching. Bouguer had proved with an elegant experiment that the eye, when used with properly designed experimental equipment and measurement procedures, could 
detect difference in brightness as little as $1.5 \%$ and thus in principle could produce rather precise measurements. By proposing the principle of simultaneous comparison, which addressed a very important condition for the proper use of the eye, Bouguer laid the foundation for visual photometry. But Bouguer failed to use the eye properly in many other respects. For example, he did not adjust the brightness of the matching fields constantly during observation; he failed to control the contrast between the matching fields and the background; and there was a large separation between the matching fields in his photometers.

All of Bouguer's successors accepted the simultaneous-comparison principle, and they worked hard to search for new experimental designs and procedures to ensure that the eye would be used properly in comparison of brightness. Herschel invented several "remote-control" devices that made constant adjustments in the brightness of the matching fields possible and thus maintained the sensibility of the eye. Potter introduced a design and procedure to avoid eye fatigue associated with contrast. Herschel's and Potter's concerns about the eye were shared by their successors Conroy and Rayleigh either used some kind of "remote-control" devices or employed an assistant to make constant adjustments, and they successfully reduced eye fatigue associated with contrast by employing an enclosed photometric head. Finally, Conroy and Rayleigh developed devices and procedures to satisfy the requirement of parallelism. Conroy invented a new photometric head that eliminated the gap between the matching fields. Rayleigh found the optimal distance between the matching fields and observer that could satisfy the requirement of parallelism while offering a proper angle of view and a suitable level of brightness.

The practitioners of visual photometry were convinced that the eye was reliable and capable of making accurate judgments in comparing brightness. They demonstrated their belief by searching for the optimal conditions for using the eye in photometric experiments. The successes of visual photometry in accurate measurements were rooted in a body of practice, which included both instrumental designs and experimental procedures, and which aimed at maintaining the eye's sensibility in comparing brightness. Based on the instrumental designs and experimental procedures developed by Bouguer, Herschel, Potter, Conroy, Rayleigh, and other practitioners, visual photometry overcame the obstacles associated with the physiological and psychological aspects of using the eye. By the beginning of the 20th century, for example, Ernst Hagen and Heinrich Rubens were able to achieve measurements of reflective power that were consistent both with theoretical calculations and with results found by other means. ${ }^{26}$ Hagen and Rubens's work and many other measurements achieved by visual photometry during this period were quickly accepted by the practitioners of physical optics, who began to use photometric measurements to test optical theories of metallic reflection and to calibrate optical constants such as the refractive indices and absorption coefficients of metals. Later, photometric measurements of reflective power were included in various reference books and became valid data. ${ }^{27}$ The legitimacy of visual photometry, which was questioned in its early years, no longer is an issue today. 


\section{Acknowledgments}

I thank Roger H. Stuewer for his careful editorial work on my paper. I also thank Gus Aldana and Bryan Card for their comments.

\section{References}

1 John Herschel, "Light," in Peter Barlow, ed., Encyclopaedia Metropolitana (London: Griffin, 1854), p. 349.

2 Xiang Chen, Instrumental Traditions and Theories of Light: The Uses of Instruments in the Optical Revolution (Dordrecht: Kluwer, 2000), pp. 99-104.

3 Pierre Bouguer, Optical Treatise on the Gradation of Light; reprint (Toronto: University of Toronto Press, 1961), p. 46

4 Ibid, p. 48.

$5 \quad$ Ibid, pp. 36-37.

$6 \quad$ Ibid, pp. 93-99.

7 Johann Lambert, Photometria, Sive, De Mensura et Gradibus Luminis, Colorum et Umbrae (Augustae Vindelicorum: V. E. Klett, 1760).

8 William Herschel, "On the Power of Penetrating into Space by Telescopes; with a Comparative Determination of the Extent of That Power in Natural Vision, and in Telescopes of Various Sizes and Constructions; Illustrated by Select Observations." Philosophical Transactions of the Royal Society of London 90 (1800), 49-64.

$9 \quad$ Ibid, pp. 64-65.

10 William Herschel, "Experiments on the Solar and on the Terrestrial Rays That Occasion Heat, with a Comparative View of the Laws to Which Light and Heat, or Rather the Rays Which Occasion Them, Are Subject, in Order to Determine Whether They Are the Same or Different," Philosophical Transactions of the Royal Society of London 90 (1800), 528.

11 Wilbur Stine, Photometrical Measurements and Manual for the General Practice of Photometry; with Especial Reference to the Photometry of Arc and Incandescent Lamps (New York: The Macmillan company, 1900), p. 76.

12 Herschel, "Experiments on the Solar" (ref. 10), p. 532

13 Ibid, p. 531.

14 Richard Potter, "An Account of Experiments to Determine the Quantity of Light Reflected by Plane Metallic Specula Under Different Angles of Incidence," The Edinburgh Journal of Science 3 (1830), 279

15 Ibid, p. 286.

16 Richard Potter, "Experiments Relating to the Reflective Powers of Crown, Plate, and Flint Glass, with Theoretical Considerations," Edinburgh Journal of Science 4 (1831), 323.

17 Chen, Instrumental Traditions and Theories of Light (ref. 2), pp. 181-183.

18 John Conroy, "Some Experiments on Metallic Reflection. No. III. On the Amount of Light Reflected by Metallic Surfaces," Proceedings of the Royal Society of London 35 (1883), 26.

19 Ibid, pp. 32-34.

20 Ogden Rood, "Photometric Experiments. Part Second. On the Amount of Light Transmitted by Plate of Polished Crown Glass at a Perpendicular Incidence," American Journal of Science 50 (1870), 1-10.

21 Lord Rayleigh (John William Strutt), "On the Intensity of Light Reflected from Certain Surfaces at Nearly Perpendicular Incidence," in Lord Rayleigh, ed., Scientific Papers, Vol. 2; reprint (New York: Dover, 1964), pp. 525-527.

22 Edward Pickering, "Measurements of the Polarization of the Light Reflected by the Sky, and by One or More Plates of Glass," Philosophical Magazine 47 (1874), 127-143.

23 John Walsh, Photometry (New York: Van Nostrand, 1926), p. 62.

24 Rayleigh, "On the Intensity of Light" (ref. 21), p. 530. 
25 Ibid., pp. 537-538.

26 Ernst Hagen and Heinrich Rubens, "Das Reflexionsvermögen von Metallen und Belegten Glasspiegeln," Annalen der Physik 1 (1900), 352-375; "Das Reflexionsvermögen einiger Metalle für Ultraviolette und Ultrarote Strahlen," Annalen der Physik 8 (1902), 1-21.

27 National Research Council, International Critical Tables of Numerical Data, Physics, Chemistry and Technology (New York: McGraw Hill, 1926-1930).

Department of Philosophy

California Lutheran University

Thousand Oaks, CA 91360-2787 USA

e-mail: chenxi@clunet.edu

\section{How to Clean a Spectroscope}

William Seabrook, Robert W. Wood's biographer, tells the following story illustrating Wood's ingenuity at his summer home on Long Island:

The spectroscope had a long wooden tube, forty-two feet in length and six inches or so in diameter, projecting out through the side of the barn, to an iron post in the cowyard, fitted at one end with a diffraction grating and at the other with a slit and a mirror. During the first winter and spring after its construction, the spiders got in and wove their webs. When Wood came down in June he spied the arachnean invasion. He grabbed the family cat and stuck it - not without a struggle - into one end of the tube, which he then closed up. Pussy, having no alternative, squirmed her way through the tunnel towards the daylight and bounded out at the other end trailing a bridal veil of spiderwebs over the fence and across the lawn. It hadn't occurred to the Professor that it would be long remembered, though he casually mentioned the episode in a technical paper in the Philosophical Magazine.

William Seabrook, Doctor Wood: Modern Wizard of the Laboratory (New York: Harcourt, Brace, 1941), p. 144. 